\title{
Influence of Enhanced Recovery Pathway on Surgical Site Infection after Colonic Surgery
}

\author{
Caroline Gronnier, ${ }^{1}$ Fabian Grass, ${ }^{1}$ Christiane Petignat, ${ }^{2}$ Basile Pache, ${ }^{1}$ Dieter Hahnloser, ${ }^{1}$ \\ Giorgio Zanetti, ${ }^{2}$ Nicolas Demartines, ${ }^{1}$ and Martin Hübner ${ }^{1}$ \\ ${ }^{1}$ Department of Visceral Surgery, Lausanne University Hospital (CHUV), Lausanne, Switzerland \\ ${ }^{2}$ Department of Hospital Preventive Medicine, Lausanne University Hospital (CHUV), Lausanne, Switzerland \\ Correspondence should be addressed to Nicolas Demartines; demartines@chuv.ch
}

Received 28 July 2017; Accepted 8 October 2017; Published 31 October 2017

Academic Editor: Fernando A. M. Herbella

Copyright (c) 2017 Caroline Gronnier et al. This is an open access article distributed under the Creative Commons Attribution License, which permits unrestricted use, distribution, and reproduction in any medium, provided the original work is properly cited.

\begin{abstract}
Background. The present study aimed to evaluate a potential effect of ERAS on surgical site infections (SSI). Methods. Colonic surgical patients operated between May 2011 and September 2015 constituted the cohort for this retrospective analysis. Over 100 items related to demographics, surgical details, compliance, and outcome were retrieved from a prospectively maintained database. SSI were traced by an independent National surveillance program. Risk factors for SSI were identified by univariate and multinomial logistic regression. Results. Fifty-four out of 397 patients (14\%) developed SSI. Independent risk factors for SSI were emergency surgery (OR 1.56; 95\% CI 1.09-1.78, $p=0.026)$, previous abdominal surgery (OR 1.7; 95\% CI 1.32-1.87, $p=0.004$ ), smoking (OR 1.71; 95\% CI 1.22-1.89, $p=0.014$ ), and oral bowel preparation (OR 1.86; 95\% CI 1.34-1.97, $p=0.013$ ), while minimally invasive surgery (OR $0.3 ; 95 \%$ CI $0.16-0.56, p<0.001)$ protected against SSI. Compliance to ERAS items of $>70 \%$ was not retained as a protective factor for SSI after multivariate analysis (OR 0.94; 95\% CI 0.46-1.92, $p=0.86$ ). Conclusions. Smoking, open and emergency surgery, and bowel preparation were risk factors for SSI. ERAS pathway had no independent impact while minimally invasive approach did. This study was registered under ResearchRegistry.com (UIN researchregistry2614).
\end{abstract}

\section{Introduction}

Enhanced recovery after surgery (ERAS) pathways aim to reduce surgical stress [1]. In colorectal surgery, ERAS has been associated with decreased complication rates, positively influencing length of stay and costs [2, 3]. Thus, ERAS represents a fundamental shift in perioperative care.

Surgical site infections (SSI) occur in up to $22 \%$ of patients and depend on patient- and surgery-related factors [4]. In Switzerland, SSI rate of $18.2 \%$ after colectomy has been described recently in a large prospective surveillance study [5].

SSI increase hospital readmission rates $[6,7]$ and health costs [8] and delay adjuvant chemotherapy [9]. Appropriate choice and timing of antibiotic prophylaxis, normothermia, and perioperative normoglycemia have been identified as protective factors against SSI $[10,11]$. Several implementation programs for evidence-based practice have demonstrated a decrease of SSI applying these principles in colorectal surgery $[12,13]$, and these items are part of ERAS guidelines for colorectal surgery [3].

The aim of the present study was to assess incidence and risk factors for SSI in a cohort of colonic surgery patients treated within an ERAS pathway.

\section{Methods}

2.1. Patients. All consecutively operated colonic surgical patients between May 2011 and September 2015 at the Lausanne University Hospital (CHUV) were included in the analysis. All patients were treated within a standardized 
ERAS pathway [14]. Open and laparoscopic colectomies in an elective or emergency (since April 2012) setting were included. The only exclusion criterium was documented anastomotic leakage in order to avoid confounding factor for SSI.

This study was approved by the Institutional Review Board (Commission cantonale d'éthique de la recherche sur l'être humain CER-VD, \# 2016-00991). The study was conducted according to the STROBE criteria and registered under ResearchRegistry.com (UIN researchregistry2614).

Demographic and surgical information was prospectively assessed in a dedicated database by the specialized ERAS nurse; accuracy of data entry was cross-checked by independent review during Hebdomadal audits. Data about ERASspecific perioperative care items were prospectively recorded [3]. Demographic information included age, gender, body mass index (BMI), American Society of Anesthesiologists (ASA) score, and smoking status (daily smoker versus nonsmoker) at the time of the procedure. Further recorded variables were diagnoses including the presence of malignancy, immunosuppressive treatments (i.e., steroids) by the time of the procedure, neoadjuvant chemotherapy, drug-requiring diabetes mellitus, and previous abdominal surgery. Surgical information included type of procedure (sigmoid, left, right, or total colectomy (excluding proctocolectomy) and Hartmann reversal), approach (open versus laparoscopy with conversion assigned to laparoscopy according to the intention-to-treat principle), setting (elective versus emergency within 72 hours after unplanned admission), duration, anastomotic technique (hand-sewn, circular, or linear stapler), and confection of a new stoma (both colo- or ileostomy). According to the Institutional guidelines, intravenous cefuroxime $1.5 \mathrm{~g}$ and metronidazole $500 \mathrm{mg}$ were systematically administered 60-30 min before incision. As an alternative in case of nontolerance, clindamycin $600 \mathrm{mg}$ and ciprofloxacin $400 \mathrm{mg}$ were used. Compliance to cefuroxime administration and timing of administration were analyzed.

2.2. Assessment of Compliance to ERAS Items. Overall compliance to 19 pre-, peri-, and postoperative ERAS care items was assessed and stratified with a cutoff of $70 \%$ according to the previous publications $[15,16]$. These items were preadmission patient education, no oral bowel preparation, preoperative oral carbohydrate drinks, no preoperative longacting sedative medication, thromboprophylaxis, antibiotic prophylaxis, postoperative nausea and vomiting (PONV) prophylaxis (droperidol $1 \mathrm{mg}$, ondansetron $4 \mathrm{mg}$, and bethamethasone $4 \mathrm{mg}$ ), intraoperative thoracic epidural analgesia, hypothermia prevention (active warming by air blanket), fluid administration guidance, balanced intravenous fluids $(\mathrm{ml} / \mathrm{kg} / \mathrm{h}<7)$, no prophylactic nasogastric tube, no abdominal drain, sip feeds at postoperative day (POD) 0 of $>300 \mathrm{kcal}$, sufficient oral fluids ( $>1 \mathrm{~L}$ ) at POD 0 , systematic laxatives, IV fluid administration at POD 1 of $<500 \mathrm{~mL}$, weight gain of $<1.5 \mathrm{~kg}$ at POD 1 , and mobilization at all at POD 0 .

2.3. Assessment and Classification of Surgical Site Infection. SSI data were prospectively monitored through an inhospital and postdischarge National surveillance program (systematic phone call at postoperative day (POD) 30) by an independent committee (http://www.swissnoso.ch). Methodological details of this assessment have been published previously [5]. SSI were classified according to the Center for Disease Control (CDC) National Nosocomial Infection Surveillance (NNIS) criteria into superficial incisional, deep incisional, and organ space infections [17]. Contamination class was assessed by the surgeon and classified at the end of the procedure as clean contaminated (grade II), contaminated (grade III), or infectious (grade IV). A second independent assessment (NNIS score 0-3) was performed by the surveillance committee according to NNIS criteria based on ASA score, wound class (independently assessed based on surgery reports and stratified likewise (II-IV)), and duration of surgery.

2.4. Outcomes/Study Endpoints. The primary endpoint was the rate of SSI. Uni- and multivariate risk factors for SSI were identified among demographic, surgery-related, and perioperative ERAS care items. Modifiable pre- and perioperative ERAS items, overall compliance to the ERAS pathway, readmission rates, and length of stay were compared between the two groups (SSI versus no SSI).

2.5. Statistical Analysis. Descriptive statistics for categorical variables were reported as frequency (\%), while continuous variables were reported as mean (standard deviation) or median (interquartile range). Chi-square was used for comparison of categorical variables. All statistical tests were two-sided, and a level of 0.05 was used to indicate statistical significance. Variables with $p$ values $\leq 0.05$ including potential confounding factors (ERAS compliance $>70 \%$, smoking status) were then entered into a multivariate logistic regression (based on a probit regression model) to provide adjusted estimations of the odds ratio (OR). Data analysis was performed with the Statistical Software for the Social Sciences SPSS Advanced Statistics 22 (IBM Software Group, 200 W. Madison St., Chicago, IL; 60606 USA).

\section{Results}

3.1. Patients. Out of 413 operated patients, 16 (4\%) were excluded due to endoscopically, surgically, or radiologically proven anastomotic leakage, leaving 397 patients for final analysis. Fifty-four patients (14\%) developed SSI. Of these, $21(39 \%)$ presented with incisional SSI, while $33(61 \%)$ were diagnosed with organ space infection.

Baseline characteristics and diagnoses are displayed in Table 1. Both groups (SSI versus no SSI) were comparable except for smoking status (22 versus $18 \%, p=0.051$ ) and previous abdominal surgery ( 46 versus $30 \%, p=0.005$ ).

Surgical details are illustrated in Table 2. Univariate risk factors for SSI were emergency setting (50 versus $27 \%$, $p<0.001)$ and wound class as assessed by the independent surveillance committee (III-IV: 65 versus 51\%, $p=0.015$ ), while minimally invasive surgery consisted a protective factor (41 versus $72 \%, p<0.001$ ).

3.2. ERAS Compliance and Modifiable Pre-and Perioperative ERAS Items. Two hundred and eight patients (52\%) 
TABLE 1: Baseline characteristics.

\begin{tabular}{|c|c|c|c|c|}
\hline & All patients $(n=397)$ & SSI $(n=54)$ & No SSI $(n=343)$ & $p$ \\
\hline Age (years) $($ mean \pm SD) & $64 \pm 16$ & $66 \pm 15$ & $64 \pm 16$ & 0.395 \\
\hline \multicolumn{5}{|l|}{ Gender } \\
\hline Male (\%) & $186(47)$ & $24(44)$ & $162(47)$ & 0.703 \\
\hline \multicolumn{5}{|l|}{$B M I(k g / m 2)$} \\
\hline$>25(\%)$ & $212(53)$ & $31(57)$ & $181(53)$ & 0.525 \\
\hline \multicolumn{5}{|l|}{ ASA group } \\
\hline I-II (\%) & $258(65)$ & $35(65)$ & $223(65)$ & \multirow{2}{*}{0.977} \\
\hline III-IV (\%) & $139(35)$ & $19(35)$ & $120(33)$ & \\
\hline Smoking (\%) & $75(19)$ & $12(22)$ & $63(18)$ & 0.051 \\
\hline Diabetes (\%) & $48(13)$ & $6(11)$ & $42(12)$ & 0.765 \\
\hline Immunosuppression (\%) & $47(12)$ & $5(9)$ & $42(12)$ & 0.528 \\
\hline Neoadjuvant chemotherapy (\%) & $26(7)$ & $3(6)$ & $24(7)$ & 0.696 \\
\hline Previous abdominal surgery (\%) & $129(33)$ & $25(46)$ & $104(30)$ & 0.005 \\
\hline Malignancy (\%) & $210(53)$ & $35(65)$ & $175(51)$ & 0.215 \\
\hline \multicolumn{5}{|l|}{ Diagnosis (\%) } \\
\hline Primary adenocarcinoma & $179(45)$ & $18(33)$ & $161(47)$ & 0.491 \\
\hline Other primary malignancy & $7(2)$ & $1(2)$ & $6(2)$ & \\
\hline Metastatic disease & $13(3)$ & $4(7)$ & $9(2)$ & \\
\hline Benign tumor/polyp & $22(6)$ & $2(4)$ & $20(6)$ & \\
\hline Inflammatory bowel disease & $26(6)$ & $5(9)$ & $21(6)$ & \\
\hline Diverticular disease & $90(23)$ & $15(28)$ & $75(22)$ & \\
\hline Functional disorder & $28(7)$ & $4(7)$ & $24(7)$ & \\
\hline Other benign disorder & $32(8)$ & $5(10)$ & $27(8)$ & \\
\hline
\end{tabular}

SSI: surgical site infection; BMI: body mass index; ASA: American Society of Anaesthesiologists. Significant values are indicated in bold characters.

presented an overall compliance of at least $70 \%$ to ERAS items. Compliance of $>70 \%$ was linked to an apparent decreased SSI rate, which did not reach the limit of significance after univariate analysis (41 versus $54 \%, p=0.065$ ). Modifiable pre- and perioperative ERAS items are illustrated in Figure 1. All items were comparable between the two groups except for oral bowel preparation (7 versus $1 \%, p=0.014)$.

Cefuroxim/metronidazole administration within one hour of incision was comparable between the two groups (63 versus $72 \%, p=0.174$ ).

3.3. Independent Risk Factors for SSI. Independent risk factors for SSI were emergency surgery (OR 1.56; 95\% CI $1.09-1.78, p=0.026$ ), previous abdominal surgery (OR 1.7; 95\% CI 1.32-1.87, $p=0.004$ ), smoking (OR 1.71; 95\% CI $1.22-1.89, p=0.014$ ), and oral bowel preparation (OR 1.86; $95 \%$ CI $1.34-1.97, p=0.013)$, while minimally invasive surgery (OR 0.3 ; 95\% CI $0.16-0.56, p<0.001$ ) protected against SSI. Compliance to ERAS items of $>70 \%$ was not a protective factor for SSI after multivariate analysis (OR 0.94; 95\% CI $0.46-1.92, p=0.86)$. Figure 2 gives an overview of independent factors associated with SSI.

3.4. Outcome. SSI were associated with longer mean length of hospital stay $(20 \pm 17$ versus $7 \pm 6$ days, $p<0.001)$ and higher readmission rate $(24$ versus $2 \%, p<0.001$ ).

\section{Discussion}

In the present study of 397 colorectal patients, it was not possible to demonstrate a benefit of ERAS compliance on SSI incidence, while minimally invasive surgery was clearly protective.

Fourteen percent of patients after colectomy presented with SSI within a 30-day postoperative observation period by an independent National surveillance committee. Several risk factors including smoking, emergency surgery, open surgery, and previous abdominal surgery were independently associated with SSI. According to ERAS guidelines, oral bowel preparation was not recommended for colonic surgery and was carried out in only $2 \%$ of the present cohort almost exclusively in the early study period. Interestingly, this was the only modifiable ERAS item independently associated with an increased SSI rate. Overall compliance to ERAS pathway of $>70 \%$ was not linked to a significant decrease in SSI neither in uni- nor in multivariate analysis.

A recent meta-analysis assessed the impact of enhanced recovery protocols on healthcare-related infections in patients undergoing abdominal and pelvic surgeries [18]. Interestingly, out of 36 included randomized controlled trials, only one single study observed a beneficial effect of ERAS. However, meta-analysis of pooled data led to a risk reduction of $25 \%$ comparing ERAS to traditional care. This finding joins formerly described beneficial effects of ERAS compared 
TABLe 2: Surgical details.

\begin{tabular}{|c|c|c|c|c|}
\hline & All patients $(n=397)(\%)$ & SSI $(n=54)(\%)$ & No SSI $(n=343)(\%)$ & $p$ \\
\hline \multicolumn{5}{|l|}{ Procedure } \\
\hline Left colectomy & $46(12)$ & $7(13)$ & $39(11)$ & \multirow{6}{*}{0.519} \\
\hline Sigmoid resection & $140(35)$ & $17(32)$ & $123(36)$ & \\
\hline Right colectomy & $135(34)$ & $21(39)$ & $114(33)$ & \\
\hline Total colectomy & $28(7)$ & $5(9)$ & $23(7)$ & \\
\hline Hartmann reversal & $39(10)$ & $4(7)$ & $35(10)$ & \\
\hline Other & $9(2)$ & 0 & $9(3)$ & \\
\hline Minimally invasive surgery & $270(68)$ & $22(41)$ & $248(72)$ & $<0.001$ \\
\hline Emergency & $118(30)$ & $27(50)$ & $91(27)$ & $<0.001$ \\
\hline Operating time $>3$ hours & $186(47)$ & $28(52)$ & $158(46)$ & 0.465 \\
\hline \multicolumn{5}{|l|}{ New stoma } \\
\hline No & $340(86)$ & $50(92)$ & $290(85)$ & \multirow{3}{*}{0.293} \\
\hline Ileostomy & $28(7)$ & $2(4)$ & $26(7)$ & \\
\hline Colostomy & $29(7)$ & $2(4)$ & $27(8)$ & \\
\hline \multicolumn{5}{|l|}{ Anastomotic technique } \\
\hline Hand-sewn & $74(18)$ & $10(19)$ & $64(19)$ & \multirow{4}{*}{0.73} \\
\hline Circular staplers & $177(45)$ & $21(39)$ & $156(46)$ & \\
\hline Linear staplers & $103(26)$ & $18(33)$ & $85(25)$ & \\
\hline Other/no anastomosis & $43(11)$ & $5(9)$ & $38(8)$ & \\
\hline \multicolumn{5}{|c|}{ Contamination class (assessed by surgeon) } \\
\hline II & $351(88)$ & $50(92)$ & $301(88)$ & \multirow{2}{*}{0.312} \\
\hline III-IV & $46(12)$ & $4(8)$ & $42(12)$ & \\
\hline \multicolumn{5}{|c|}{ Wound class (assessed by surveillance committee) } \\
\hline II & $187(47)$ & $19(35)$ & $168(49)$ & \multirow{2}{*}{0.015} \\
\hline III-IV & $210(53)$ & $35(65)$ & $175(51)$ & \\
\hline \multicolumn{5}{|l|}{ Antibiotic administration } \\
\hline$<1$ hour before incision & $281(71)$ & $34(63)$ & $247(72)$ & 0.174 \\
\hline \multicolumn{5}{|l|}{ NNIS score } \\
\hline 0 & $74(19)$ & $7(13)$ & $67(20)$ & \multirow{4}{*}{0.059} \\
\hline 1 & $145(37)$ & $15(28)$ & $130(38)$ & \\
\hline 2 & $138(35)$ & $22(41)$ & $116(34)$ & \\
\hline 3 & $40(10)$ & $10(19)$ & $30(9)$ & \\
\hline
\end{tabular}

SSI: surgical site infection; Contamination/wound class: II—clean contaminated, III—contaminated, IV—infectious; NNIS: National Nosocomial Infection Surveillance. Significant values are indicated in bold characters.

to traditional care. Reduced overall morbidity, length of stay, and costs have been repeatedly proven as a result of decreased surgical stress response $[2,14,19]$. In the present study, all patients without exception were treated within a standardized ERAS care pathway, including emergency operations. The aim was thus not to compare SSI rate to traditional care but to assess SSI rate among different compliance groups. Gustafsson has shown that best results were achieved with the highest overall compliance to ERAS [15]. These results have been confirmed in a recent study suggesting a minimal overall compliance of $70 \%$ to achieve improved postsurgical results [16]. As a consequence, the present study aimed to compare compliant $(>70 \%)$ to less compliant $(<70 \%)$ patients and to analyze individual compliance to modifiable ERAS items. Two reasons might account for similar SSI rates among compliant and less compliant patients. First, the modest sample size might be prone to type II error. Second, ERAS has been shown to decrease above all nonsurgical, especially cardiopulmonary complications, rather than surgical complications [2].

In the present cohort, $14 \%$ of patients were diagnosed with either superficial or organ space SSI as defined. This rate is comparable to former large-scale studies [20-22]. It has to be emphasized that all patients were systematically followed by dedicated and independent abstractors limiting artificially low infection rates due to detection bias.

Surgical complications and anastomotic leaks are mainly linked to patient-related factors or caused by technical shortcomings. Therefore, surgical complications were shown not to be modified by ERAS pathways [2]. Inevitably, anastomotic leaks entail intra-abdominal abscess and frequently superficial side infection as a consequence. Hence, patients 


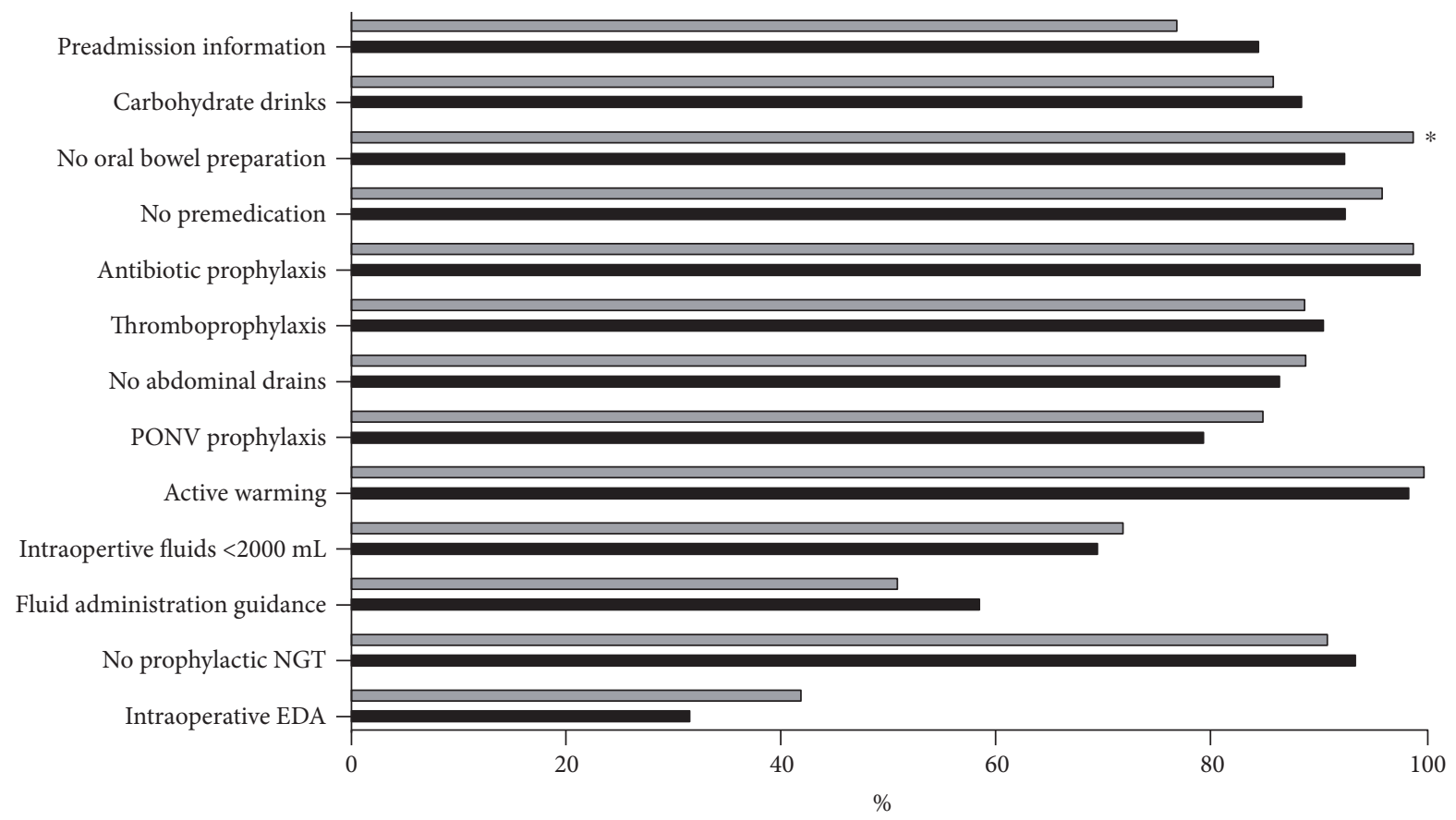

No SSI

SSI

FIgURE 1: Pre- and intraoperative modifiable ERAS items. Comparison of compliance to modifiable pre- and intraoperative ERAS-related items among patients with SSI (black bars) and patients without SSI (grey bars). Premedication = administration of long-acting sedative medication. SSI: surgical site infection; PONV: postoperative nausea and vomiting; EDA: epidural analgesia; NGT: nasogastric tube. * indicates statistical significance $(p<0.05)$.

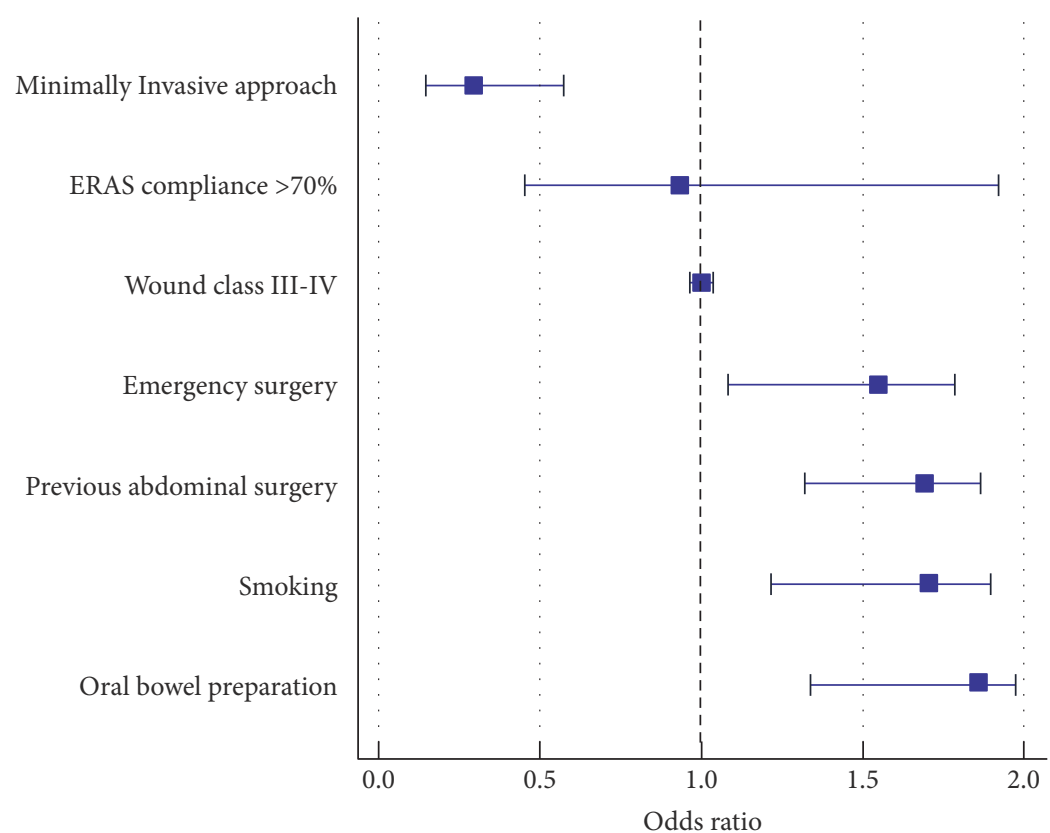

FIGURE 2: Independent risk factors for SSI. Odds ratio for outcome SSI, 95\% confidence IntervaI.

with a proven anastomotic leak were excluded as an overwhelming confounder for the purpose of the present study. However, contamination class assessed by the treating surgeon and independent assessment of perioperative contamination by the surveillance committee (NNIS score, wound class) were analyzed as potential risk factors. Despite 
significance after univariate analysis, wound class did not correlate with SSI after multivariate analysis. An explanation might be that both measures rely on subjective assessment, and hence observer-dependent differences in individual grading of contamination might be a consequence. Interestingly, surgeons classified most procedures (88\%) as contamination class II, while half of the procedures were classified as grades III-IV by the independent committee. Another parameter that deserves in-depth discussion is antimicrobial prophylaxis [23]. In the present cohort, antibiotic prophylaxis was delivered in all patients without exception, however with appropriate timing of administration between 3060 min before incision in $71 \%$ of patients only. Despite a difference of almost $10 \%$ among the two groups (63 versus $72 \%$ ), this result was not significant, possibly due to type II error. While timing of antibiotic administration is crucial, mode of administration is debated [24]. While a combined oral and IV administration has been most beneficial in a former study [25], no superiority of this combination was observed in a more recent randomized trial [26]. Due to an inherent risk of dehydration, distress and postoperative ileus, mechanical bowel preparation is not recommended by ERAS guidelines [3], in line with a comprehensive Cochrane review of almost 6000 patients [27], and should remain an exception, that is, when intraoperative colonoscopy is needed. Whether oral antibiotics need to be combined with mechanical bowel preparation is a matter of debate [24]. Recent evidence suggested this combination [28-30] as a way to reduce SSI, in contrast to the results of former randomized studies [31]. To draw final conclusions, results of ongoing randomized trials (i.e., MOBILE trial, NCT02652637) are eagerly awaited before the next revision of enhanced recovery guidelines for colorectal surgery.

In a large quality improvement project including more than 27000 patients who underwent colonic surgery, open approach and active smoking were independent risk factors for both superficial and deep/organ space infection [32], similar to the results of the present study. It is therefore important to insist during the outpatient visit on smoking cessation prior to surgery. Minimally invasive colonic surgery was associated with a lower rate of SSI in several studies [33, 34]. Moreover, the combination of laparoscopy and ERAS might be even more beneficial [35-37]. Several known risk factors for SSI, including diabetes and hypothermia, were not retained in the present study $[38,39]$, probably reflecting benefits of the ERAS protocol including stringent carbohydrate homeostasis and high compliance to hypothermia prevention.

Some limitations of the present study beyond its retrospective nature need to be discussed. Due to modest sample size, the study is prone to type II error. Some of the analyzed items (wound/contamination class and NNIS score) rely on subjective assessment. Moreover, it is delicate to compare SSI rate to previous reports since definition of SSI was not standardized among studies. However, the strength of the present study was independent and prospective assessment of SSI in all patients by the official National surveillance committee. Thus, the results are likely to be representative for everyday practice for physicians performing colonic surgery within an ERAS program.

\section{Conclusions}

In conclusion, SSI remain a frequent problem after colonic surgery. While ERAS compliance had no independent impact, open approach, emergency surgery, smoking, and bowel preparation were identified as independent risk factors for SSI. Preoperative smoking cessation and favoring of minimally invasive surgery might contribute to better outcomes.

\section{Disclosure}

This study was presented at the 5th ERAS World Congress, May 10-12, Lyon, France and at the Annual Swiss Surgical Meeting 2017, May 31-June 2, Bern Switzerland.

\section{Conflicts of Interest}

The authors declare that there is no conflict of interest regarding the publication of this article.

\section{Authors' Contributions}

Caroline Gronnier and Fabian Grass shared first authorship.

\section{Acknowledgments}

The authors would like to acknowledge the support provided by all members of the enhanced recovery after surgery team in Lausanne, in particular Valérie Addor, dedicated ERAS nurse.

\section{References}

[1] M. J. Scott, G. Baldini, K. C. Fearon et al., "Enhanced recovery after surgery (ERAS) for gastrointestinal surgery, part 1: pathophysiological considerations," Acta Anaesthesiologica Scandinavica, vol. 59, no. 10, pp. 1212-1231, 2015.

[2] M. Greco, G. Capretti, L. Beretta, M. Gemma, N. Pecorelli, and M. Braga, "Enhanced recovery program in colorectal surgery: a meta-analysis of randomized controlled trials," World Journal of Surgery, vol. 38, no. 6, pp. 1531-1541, 2014.

[3] U. O. Gustafsson, M. J. Scott, W. Schwenk et al., "Guidelines for perioperative care in elective colonic surgery: enhanced recovery after surgery (ERAS ${ }^{\circledR}$ ) society recommendations," World Journal of Surgery, vol. 37, no. 2, pp. 259-284, 2013.

[4] S. S. Forbes, W. J. Stephen, W. L. Harper et al., "Implementation of evidence-based practices for surgical site infection prophylaxis: results of a pre- and postintervention study," Journal of the American College of Surgeons, vol. 207, no. 3, pp. 336-341, 2008.

[5] W. Staszewicz, M. C. Eisenring, V. Bettschart, S. Harbarth, and N. Troillet, "Thirteen years of surgical site infection surveillance in Swiss hospitals," Journal of Hospital Infection, vol. 88, no. 1, pp. 40-47, 2014.

[6] T. G. Kerwel, S. W. Leichtle, T. Asgeirsson, S. K. Hendren, R. K. Cleary, and M. A. Luchtefeld, "Risk factors for readmission after elective colectomy: postoperative complications are more important than patient and operative factors," Diseases of the Colon \& Rectum, vol. 57, no. 1, pp. 98-104, 2014. 
[7] R. P. Merkow, M. H. Ju, J. W. Chung et al., "Underlying reasons associated with hospital readmission following surgery in the United States," JAMA, vol. 313, no. 5, pp. 483-495, 2015.

[8] E. C. Wick, A. D. Shore, K. Hirose et al., "Readmission rates and cost following colorectal surgery," Diseases of the Colon \& Rectum, vol. 54, no. 12, pp. 1475-1479, 2011.

[9] R. P. Merkow, D. J. Bentrem, M. F. Mulcahy et al., "Effect of postoperative complications on adjuvant chemotherapy use for stage III colon cancer," Annals of Surgery, vol. 258, no. 6, pp. 847-853, 2013.

[10] A. C. Melling, B. Ali, E. M. Scott, and D. J. Leaper, "Effects of preoperative warming on the incidence of wound infection after clean surgery: a randomised controlled trial," The Lancet, vol. 358, no. 9285, pp. 876-880, 2001.

[11] D. C. Classen, R. S. Evans, S. L. Pestotnik, S. D. Horn, R. L. Menlove, and J. P. Burke, "The timing of prophylactic administration of antibiotics and the risk of surgical-wound infection," The New England Journal of Medicine, vol. 326, no. 5, pp. 281-286, 1992.

[12] J. Tanner, W. Padley, O. Assadian, D. Leaper, M. Kiernan, and C. Edmiston, "Do surgical care bundles reduce the risk of surgical site infections in patients undergoing colorectal surgery? A systematic review and cohort meta-analysis of 8,515 patients," Surgery, vol. 158, no. 1, pp. 66-77, 2015.

[13] E. C. Wick, D. B. Hobson, J. L. Bennett et al., "Implementation of a surgical comprehensive unit-based safety program to reduce surgical site infections," Journal of the American College of Surgeons, vol. 215, no. 2, pp. 193-200, 2012.

[14] D. Roulin, A. Donadini, S. Gander et al., "Cost-effectiveness of the implementation of an enhanced recovery protocol for colorectal surgery," British Journal of Surgery, vol. 100, no. 8, pp. 1108-1114, 2013.

[15] U. O. Gustafsson, J. Hausel, A. Thorell et al., "Adherence to the enhanced recovery after surgery protocol and outcomes after colorectal cancer surgery," Archives of Surgery, vol. 146, no. 5, pp. 571-577, 2011.

[16] J. Jurt, J. Slieker, P. Frauche et al., "Enhanced recovery after surgery: can we rely on the key factors or do we need the Bel Ensemble?," World Journal of Surgery, vol. 41, no. 10, pp. 2464-2470, 2017.

[17] T. G. Emori, D. H. Culver, T. C. Horan et al., "National nosocomial infections surveillance system (NNIS): description of surveillance methods," American Journal of Infection Control, vol. 19, no. 1, pp. 19-35, 1991.

[18] M. C. Grant, D. Yang, C. L. Wu, M. A. Makary, and E. C. Wick, "Impact of enhanced recovery after surgery and fast track surgery pathways on healthcare-associated infections: results from a systematic review and meta-analysis," Annals of Surgery, vol. 265, no. 1, pp. 68-79, 2017.

[19] A. A. Veenhof, M. S. Vlug, M. H. van der Pas et al., "Surgical stress response and postoperative immune function after laparoscopy or open surgery with fast track or standard perioperative care: a randomized trial," Annals of Surgery, vol. 255, no. 2, pp. 216-221, 2012.

[20] A. Gomila, J. Carratala, D. Camprubi et al., "Risk factors and outcomes of organ-space surgical site infections after elective colon and rectal surgery," Antimicrobial Resistance \& Infection Control, vol. 6, no. 1, p. 40, 2017.

[21] A. Gomila, J. M. Badia, J. Carratala et al., "Current outcomes and predictors of treatment failure in patients with surgical site infection after elective colorectal surgery. A multicentre prospective cohort study," Journal of Infection, vol. 74, no. 6, pp. 555-563, 2017.

[22] S. Goto, S. Hasegawa, H. Hata et al., "Differences in surgical site infection between laparoscopic colon and rectal surgeries: sub-analysis of a multicenter randomized controlled trial (Japan-multinational trial organization PREV 07-01)," International Journal of Colorectal Disease, vol. 31, no. 11, pp. 1775-1784, 2016.

[23] S. Hendren, D. Fritze, M. Banerjee et al., "Antibiotic choice is independently associated with risk of surgical site infection after colectomy: a population-based cohort study," Annals of Surgery, vol. 257, no. 3, pp. 469-475, 2013.

[24] R. L. Nelson, E. Gladman, and M. Barbateskovic, "Antimicrobial prophylaxis for colorectal surgery," Cochrane Database of System Reviews, no. 5, article CD001181, 2014.

[25] H. Hata, T. Yamaguchi, S. Hasegawa et al., "Oral and parenteral versus parenteral antibiotic prophylaxis in elective laparoscopic colorectal surgery (JMTO PREV 07-01): a phase 3, multicenter, open-label, randomized trial," Annals of Surgery, vol. 263, no. 6, pp. 1085-1091, 2016.

[26] A. Ikeda, T. Konishi, M. Ueno et al., "Randomized clinical trial of oral and intravenous versus intravenous antibiotic prophylaxis for laparoscopic colorectal resection," British Journal of Surgery, vol. 103, no. 12, pp. 1608-1615, 2016.

[27] K. F. Guenaga, D. Matos, and P. Wille-Jorgensen, "Mechanical bowel preparation for elective colorectal surgery," The Cochrane Database of Systematic Reviews, no. 9, article CD001544, 2011.

[28] M. S. Morris, L. A. Graham, D. I. Chu, J. A. Cannon, and M. T. Hawn, "Oral antibiotic bowel preparation significantly reduces surgical site infection rates and readmission rates in elective colorectal surgery," Annals of Surgery, vol. 261, no. 6, pp. 1034-1040, 2015.

[29] A. A. Althumairi, J. K. Canner, T. M. Pawlik et al., "Benefits of bowel preparation beyond surgical site infection: a retrospective study," Annals of Surgery, vol. 264, no. 6, pp. 1051-1057, 2016.

[30] J. E. Scarborough, C. R. Mantyh, Z. Sun, and J. Migaly, “Combined mechanical and oral antibiotic bowel preparation reduces incisional surgical site infection and anastomotic leak rates after elective colorectal resection: an analysis of colectomy-targeted ACS NSQIP," Annals of Surgery, vol. 262, no. 2, pp. 331-337, 2015.

[31] B. S. Reddy, J. Macfie, M. Gatt, C. N. Larsen, S. S. Jensen, and T. D. Leser, "Randomized clinical trial of effect of synbiotics, neomycin and mechanical bowel preparation on intestinal barrier function in patients undergoing colectomy," British Journal of Surgery, vol. 94, no. 5, pp. 546-554, 2007.

[32] E. H. Lawson, B. L. Hall, and C. Y. Ko, "Risk factors for superficial vs deep/organ-space surgical site infections: implications for quality improvement initiatives," JAMA Surgery, vol. 148, no. 9, pp. 849-858, 2013.

[33] G. Gandaglia, K. R. Ghani, A. Sood et al., "Effect of minimally invasive surgery on the risk for surgical site infections: results from the National Surgical Quality Improvement Program (NSQIP) Database," JAMA Surgery, vol. 149, no. 10, pp. 1039-1044, 2014.

[34] S. Romy, M. C. Eisenring, V. Bettschart, C. Petignat, P. Francioli, and N. Troillet, "Laparoscope use and surgical site infections in digestive surgery," Annals of Surgery, vol. 247, no. 4, pp. 627-632, 2008. 
[35] M. S. Vlug, J. Wind, M. W. Hollmann et al., "Laparoscopy in combination with fast track multimodal management is the best perioperative strategy in patients undergoing colonic surgery: a randomized clinical trial (LAFA-study)," Annals of Surgery, vol. 254, no. 6, pp. 868-875, 2011.

[36] R. H. Kennedy, E. A. Francis, R. Wharton et al., "Multicenter randomized controlled trial of conventional versus laparoscopic surgery for colorectal cancer within an enhanced recovery programme: EnROL," Journal of Clinical Oncology, vol. 32, no. 17, pp. 1804-1811, 2014.

[37] F. Esteban, F. J. Cerdan, M. Garcia-Alonso et al., "A multicentre comparison of a fast track or conventional postoperative protocol following laparoscopic or open elective surgery for colorectal cancer surgery," Colorectal Disease, vol. 16, no. 2, pp. 134-140, 2014.

[38] R. S. Jackson, R. L. Amdur, J. C. White, and R. A. Macsata, "Hyperglycemia is associated with increased risk of morbidity and mortality after colectomy for cancer," Journal of the American College of Surgeons, vol. 214, no. 1, pp. 68-80, 2012.

[39] G. B. Melton, J. D. Vogel, B. R. Swenson, F. H. Remzi, D. A. Rothenberger, and E. C. Wick, "Continuous intraoperative temperature measurement and surgical site infection risk: analysis of anesthesia information system data in 1008 colorectal procedures," Annals of Surgery, vol. 258, no. 4, pp. 606-612, 2013. 


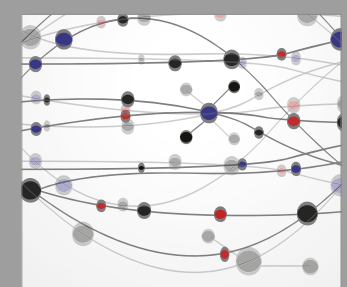

The Scientific World Journal
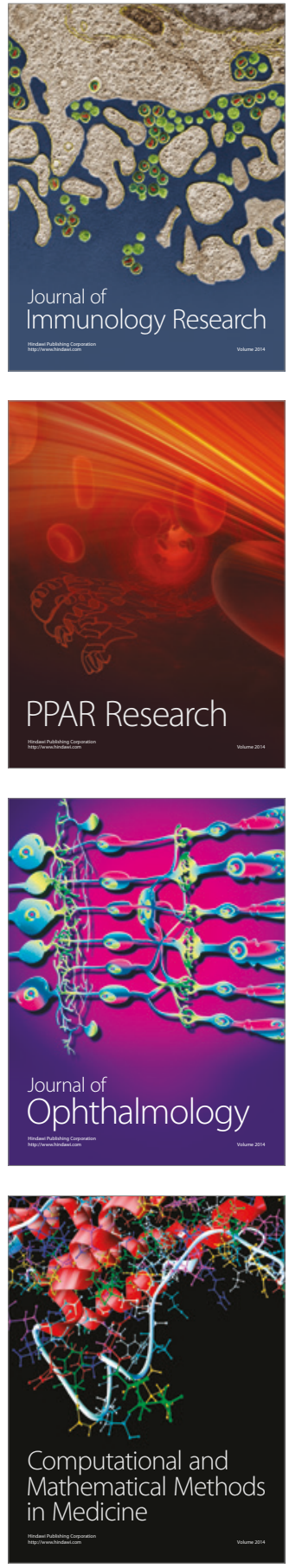

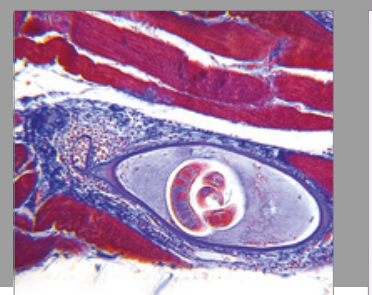

Gastroenterology Research and Practice
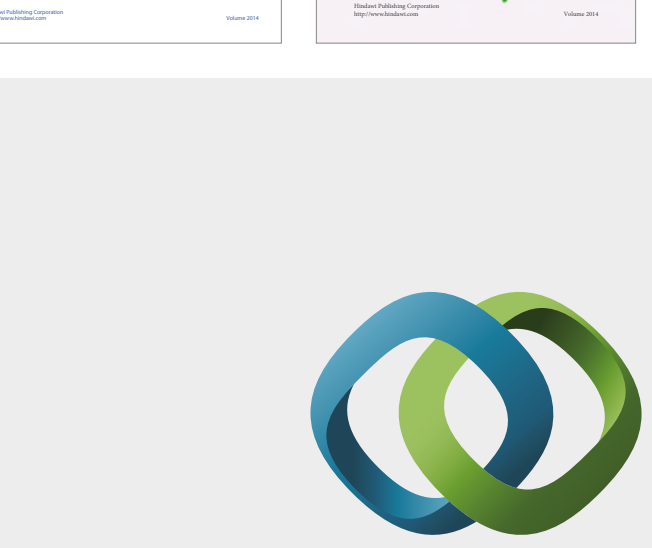

\section{Hindawi}

Submit your manuscripts at

https://www.hindawi.com
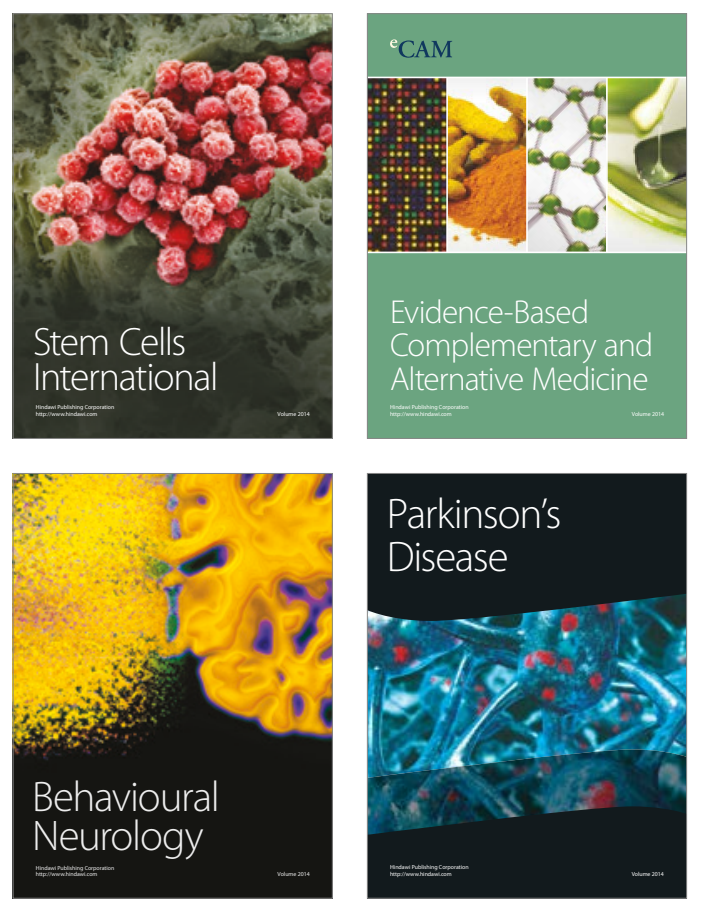
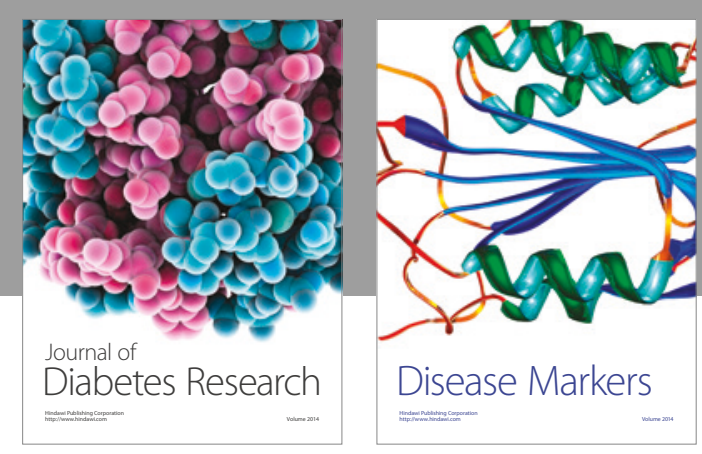

Disease Markers
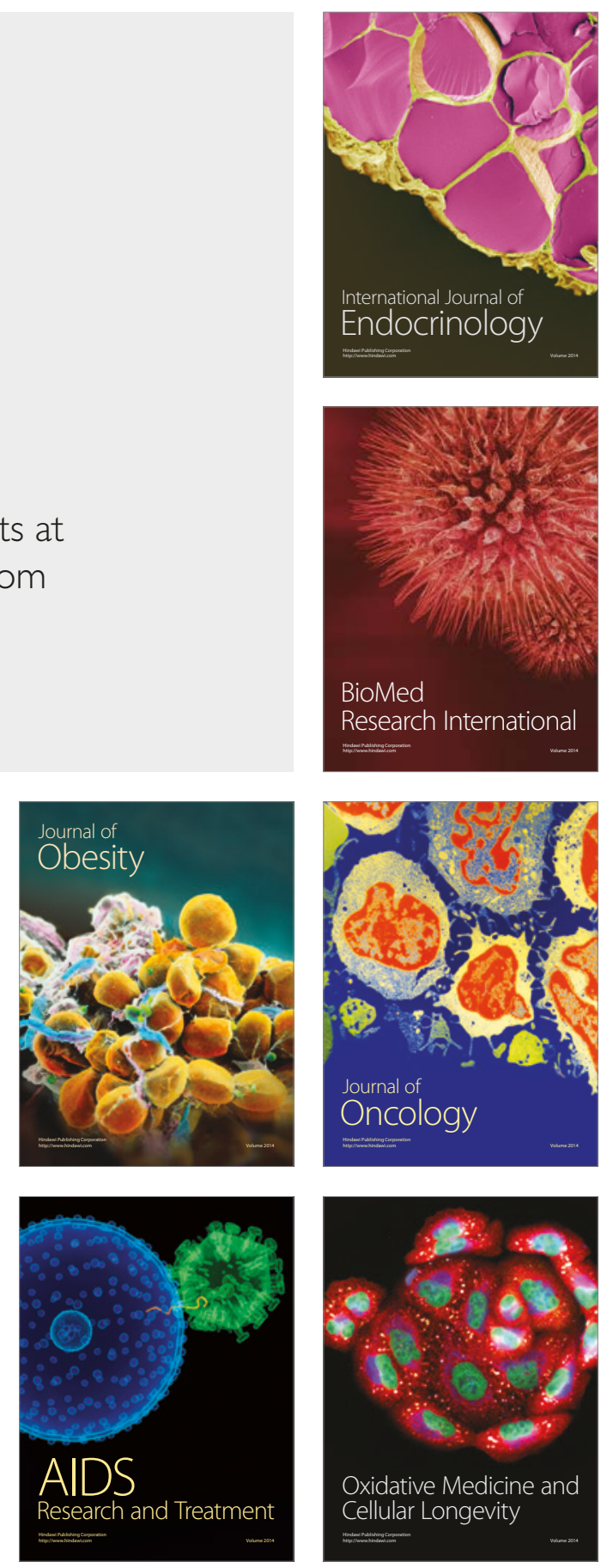